\title{
Influence of Coronary Artery Stenosis Severity and Coronary Collaterali- zation on Extent of Chronic Myocardial Scar: Insights from Quantitative Coronary Angiography and Delayed-Enhancement MRI
}

\author{
Daniel Bexell ${ }^{1,5}$, Randolph M. Setser ${ }^{1}$, Paul Schoenhagen ${ }^{1,2}$, Michael L. Lieber ${ }^{3}$, Sorin J. Brener ${ }^{2}$, \\ Thomas B. Ivanc ${ }^{2}$, Eva M. Balazs ${ }^{2}$, Thomas P. O`Donnell ${ }^{4}$, Arthur E. Stillman ${ }^{1}$, Håkan Arheden $^{5}$, \\ Galen S. Wagner ${ }^{6}$ and Richard D. White*,1,2
}

\author{
${ }^{1}$ From the Departments of Diagnostic Radiology, ${ }^{2}$ Cardiovascular Medicine and ${ }^{3}$ Quantitative Health Sciences, \\ ${ }^{4}$ Cleveland Clinic, Cleveland, OH, Siemens Corporate Research, ${ }^{5}$ Princeton, NJ, Department of Clinical Physiology, \\ ${ }^{6}$ Lund University, Lund, Sweden, and Division of Cardiology, Duke University Medical Center, Durham, NC
}

\begin{abstract}
Objectives: In patients with chronic ischemic heart disease, the relationship between coronary artery lesion severity and myocardial scarring is unknown. The purpose of this study was to examine the relationship between proximal coronary artery stenosis severity, the amount of coronary collateralization, and myocardial scar extent in the distal distribution of the affected coronary artery based on both quantitative coronary angiography (QCA) and delayed-enhancement magnetic resonance imaging (DE-MRI).

Methods: Thirty-four patients (26 males, 8 females; age range: 35-86 years) with a coronary artery containing a single, proximal stenosis $\geq 30 \%$ by quantitative coronary angiography (QCA) underwent DE-MRI. The relationship between stenosis severity, collateralization, and myocardial scar morphology (area, transmurality and patchiness) was examined using linear mixed-model ANCOVA.

Results: There was a statistically significant correlation between stenosis severity and scar extent $(\mathrm{r}=0.53, \mathrm{p}<0.01)$. Patients with hemodynamically significant stenoses $(\geq 70 \%)$ exhibited significantly greater collateralization $(\mathrm{p}<0.05)$ and scar extent $(\mathrm{p}<0.01)$ than patients with $<70 \%$ stenosis. However, scarring was often found in patients with stenoses $<70 \%$. Also, greater stenosis severity $(93 \pm 14 \%)$ and mean scar extent $(41 \pm 35 \%)$ were found in patients with collaterals than in patients without collaterals (diameter stenosis $48 \pm 10 \%, \mathrm{p}<0.01$ ) (scar extent 19 $\pm 29 \%, \mathrm{p}=0.01$ ).

Conclusions: Using QCA and DE-MRI, we demonstrate a significant relationship between coronary artery stenosis severity and myocardial scar extent, in the absence of a documented history of acute infarction. The relationship likely reflects increasing ischemia leading to scar formation in the range of angiographically significant stenosis. However, in the absence of collateralization, scar was observed without significant stenosis, especially in females.
\end{abstract}

Key Words: Coronary collateralization, coronary stenosis, magnetic resonance imaging, myocardial ischemia, myocardial scar, quantitative coronary angiography.

\section{INTRODUCTION}

The relationship between the degree of flow-limiting coronary stenosis and the resulting myocardial ischemia has been described in experimental and clinical studies [1-4]. It is recognized that repetitive, transient episodes of myocardial ischemia may lead to myocardial hibernation and eventually progressive development of myocardial scarring [5-8]. However, the relationship between lesion severity and myocardial scar development is not well known.

Because of its high spatial resolution, delayed-enhancement magnetic resonance imaging (DE-MRI) allows identification of transmural and non-transmural myocardial scar formation. Therefore, the DE-MRI technique [9] uniquely

*Address correspondence to this author at the Department of Radiology, University of Florida College of Medicine-Jacksonville, 655 West 8th Street, Jacksonville, FL 32209, USA; Tel: (904) 244-4224; Fax: (904) 2448827; E-mail: richard.white@jax.ufl.edu permits quantitative characterization of different patterns of hyperenhanced myocardial scar [10] for the aforementioned pursuit.

The purpose of this study was to examine further the relationship between the severity of proximal coronary artery stenosis, the amount of stimulated coronary collateralization, and the extent (degree and pattern) of resultant myocardial scar in the distal distribution of the affected coronary artery based on both quantitative coronary angiography (QCA) and quantitative DE-MRI. To this end, patients with known significant coronary artery disease (CAD), causing chronic ischemic heart disease, but no documented history of acute myocardial infarction, were targeted.

\section{METHODS}

\section{Study Population}

This was a retrospective observational clinical study. With prior Institutional Review Board approval, we re- 
viewed the medical records of chronic ischemic heart disease patients who underwent both a clinically indicated cardiac MRI examination, for myocardial viability assessment $[11,12]$, and relatively concurrent selective coronary angiography. The mean period between cardiac MRI and coronary angiography was $16 \pm 19$ days (range $0-84$ days). We identified 34 patients (age $67 \pm 11$ years, range 35-86 years; gender: 26 males, 8 females) with a single, focal angiographic stenosis of $\geq 30 \%$ luminal diameter involving the proximal segment of 1 of the 3 major epicardial coronary arteries. Although all patients had advanced, multi-vessel coronary artery disease, these patients had a single lesion in the artery of interest.

Based on a consensus review of the coronary angiographic images, the index lesion was defined as follows: LAD, if it occurred in the proximal segment of the left anterior descending (LAD) coronary artery, between its origin and the first major diagonal branch; LCX, if it occurred in the proximal segment of the left circumflex (LCX) artery, defined as between its origin and the first lateral branch; or PDA, if it occurred anywhere along the dominant or codominant coronary artery giving rise to the posterior descending artery (PDA), or in the proximal PDA itself. Coronary artery dominance was established by the source of both the PDA and the postero-lateral system supplying branches to the posterior and lateral regions of the left ventricle (LV); most often the right coronary artery (RCA) was dominant. Co-dominance indicated PDA origin from the RCA and the postero-lateral system from the LCX.

Exclusion criteria included $>30 \%$ narrowing more distally in the same major coronary artery of interest, or a clinically documented history of acute myocardial infarction in its distribution. Due to the potential confounding influence, patients with $\geq 30 \%$ stenosis of the left main coronary trunk excluded from the LAD and LCX groups. Patients with a history of either coronary artery bypass grafting or percutaneus transluminal coronary angioplasty/stenting of the epicardial coronary artery of interest, or a history of medical conditions pre-disposing to microvascular disease (e.g., diabetes mellitus, vasculitis) were also excluded.

\section{Magnetic Resonance Imaging}

All imaging was performed on a 1.5 Tesla MRI scanner (Symphony or Sonata, Siemens Medical Solutions, Erlangen, Germany) using a phased-array torso coil and standard ECG referencing techniques.

For evaluation of myocardial scar, LV short-axis DEMRI was performed at the mid-LV level, defined by the papillary muscle insertions, and at the basal level, defined midway between the mid-LV and mitral annulus. Apical images were not included for analysis because of concerns over cumulative effects of more distal coronary atherosclerosis. DEMRI images were acquired using an inversion recovery spoiled gradient echo protocol 20-30 minutes after intravenous injection of Gadolinium-DTPA (0.2 mmol per kilogram) [9]. Sequence parameters were FOV 280-350 mm, acquisition matrix 256x161, slice thickness 5-10 mm; TE 4.0 msec, TR sequence $8 \mathrm{msec}$, effective $2 \mathrm{RR}$ intervals, flip angle $30^{\circ}$, and TI $175-275 \mathrm{msec}$ to null signal from viable myocardium in late diastole following an appropriate delay after the R-wave trigger. This imaging was performed with
(1 acquisition) or without (2-3 acquisitions) breath-holding, based on heart rate and ability to suspend respiration.

Dynamic cine image-loops were acquired in contiguous short-axis slices covering the heart from the mitral valve to the LV apex. Cine images were acquired using a segmented $\mathrm{k}$-space balanced steady state free precession (bSSFP) protocol: FOV 280-350 mm, matrix 256x120, slice thickness 8-10 $\mathrm{mm}$, TR $4 \mathrm{msec}$, TE $1.6 \mathrm{msec}$. Fifteen lines per segment were acquired for a temporal resolution of $32 \mathrm{msec}$ with 15 27 temporal phases covering the cardiac cycle. Imaging at each anatomic level was performed with (1 acquisition) or without (2-3 acquisitions) breath-holding.

\section{Quantitative Coronary Angiography for Coronary Stenosis Evaluation}

QCA $[13,14]$ was performed from multiple orthogonal views using standard technique in an established core laboratory at our institution. The angiograms were evaluated with the CAAS II System QCA edge-detection software (Pie Medical Inc., Maastricht, The Netherlands) following standard core lab procedures. A cine viewer (Vanguard XR-35) was utilized for cases received on $35 \mathrm{~mm}$ cine film and the CAAS II dicom viewer was used to review digital cases. The following data were recorded for each coronary artery segment: percent diameter reduction of the segment, percent area reduction of the segment, and the minimum lesion diameter.

\section{Quantitative Collateral Circulation Grading}

The extent of collateralization to the coronary artery of interest was described using two separate scoring approaches, as follows:

1. Rentrop Score: 0 = no visible collateralization, $1=$ faint filling of only side branches of coronary artery of interest, 2 = partial filling of artery of interest, and $3=$ complete filling of artery of interest. The reproducibility of this grading has been validated previously $[13,14]$.

2. Collateral Score: This scoring approach represents the number of coronary artery systems, including that of the artery of interest itself (by bridge collaterals), contributing collateral branches to the dependent myocardial region of interest as follows: $0=$ no contributor, $1=1$ contributor, $2=2$ contributors, and $3=3$ contributors.

\section{Quantitative Analysis of DE-MRI for Scar Charac- terization}

An observer blinded to the results of QCA performed the quantitative MRI analysis using a previously described quantitative approach with low inter-observer variability [10]. In basal and mid-ventricular DE-MRI images, the myocardium was delineated manually (Argus, Siemens Medical Systems, Erlangen, Germany) while referencing spatially and temporally corresponding cine images. Evaluation of scar distribution was then performed on the same workstation using prototype software (modified Argus, Siemens Corporate Research, Princeton, NJ). The user initially outlined scar regions and then visually determined optimal thresholds to differentiate viable myocardium from myocardial scar within that region of interest using an interactive region-filling tool, as previously described [10]. 
At each of these 2 short-axis levels, quantitative analysis of thresholded images was restricted to a $30^{\circ}$ myocardial sector straddling segments representative of the distribution of a particular major coronary artery [15]; the coronary artery of interest was centered over this sector (Fig. (1)). The myocardial sector was centered below the visualized LAD course in the anterior interventricular groove for LAD lesions, the mid-lateral free-wall (centered opposite the midinterventricular septum) for lesions in the LCX, or the visualized PDA course in the posterior interventricular groove for proximal lesions in either the source artery of the PDA or the proximal PDA itself.

Quantitative analysis of thresholded images (Matlab, MathWorks, Natick, MA) was then performed to calculate the following parameters [10], expressed relative to the sector of myocardium underlying the coronary artery of interest:

1. Scar Area: The relative area of non-viable myocardium within a sector, computed as the ratio of non-viable pixels (white) within the myocardium to total pixels (white+black) within the myocardial sector.

2. Transmurality: The proportion of radial chords per sector with scar transmurality traversing the endocardial to epicardial extent $\geq 50 \%$.

3. Patchiness: A measure of the coherence of non-viable myocardium within the sector, computed as the ratio of non-viable edge pixels to total non-viable pixels within each thresholded sector, where edge pixels were defined as white pixels which border at least one viable pixel (black) in a 8-connected neighborhood [16].

\section{Statistical Methods}

The strength of association between stenosis severity (percent diameter and percent area) and each scar parameter (scar area, transmurality and patchiness), before controlling for the effects of confounding factors, was assessed using Pearson product-moment correlation. The statistical significance of differences between pairs of independent groups was assessed using the Mann-Whitney test.
Linear mixed-model analysis of covariance (ANCOVA) methods [17] were used to assess the significance of percent diameter stenosis, percent area stenosis and collateral score in explaining the variation in each scar parameter (percent scar, percent transmurality and patchiness). Each scar parameter was treated as a separate outcome in three distinct linear mixed-models. Other covariates included in each model were gender, age, vessel type (e.g. LAD), anatomic level (e.g. basal) and Rentrop score.

After the statistical significance of predictors and covariates was tested in each model, the fitted model was used to predict scar outcomes based on hypothetical values for the set of variables in each model. In determining the appropriate value to use for percent area stenosis, given a specific percent diameter stenosis, we made use of a regression equation that approximated the (quadratic) relationship between the two. In deciding which hypothetical value to use for a variable such as age, the mean was used (i.e., mean age of all patients). For categorical variables such as vessel type or level, the weighted mean effect of all categories of each such variable was used in the fitted model.

\section{RESULTS}

The location of the index proximal coronary artery lesion was distributed as follows: 6 LAD, 4 LCX, 24 PDA. The PDA group included 23 patients with RCA dominance and 1 patient with left coronary artery dominance. The characteristics of the patient population overall, as well as individually for the LAD, LCX and PDA groups, are shown in Table $\mathbf{1}$. There was no statistically significant difference among the vessel groups with respect to age $(\mathrm{p}=0.49)$, percent diameter stenosis $(\mathrm{p}=0.98)$, percent area stenosis $(\mathrm{p}=0.98)$, Rentrop score $(p=0.60)$ or collateral score $(p=0.66)$. Furthermore, there was no significant correlation between age and myocardial scar extent $\left(\mathrm{R}^{2}=0.03, \mathrm{p}=0.64\right)$.

\section{Relationship Between Coronary Stenosis Severity, Collateralization and Myocardial Scarring}

Correlations between the degree of coronary artery stenosis and each myocardial scar parameter were statistically

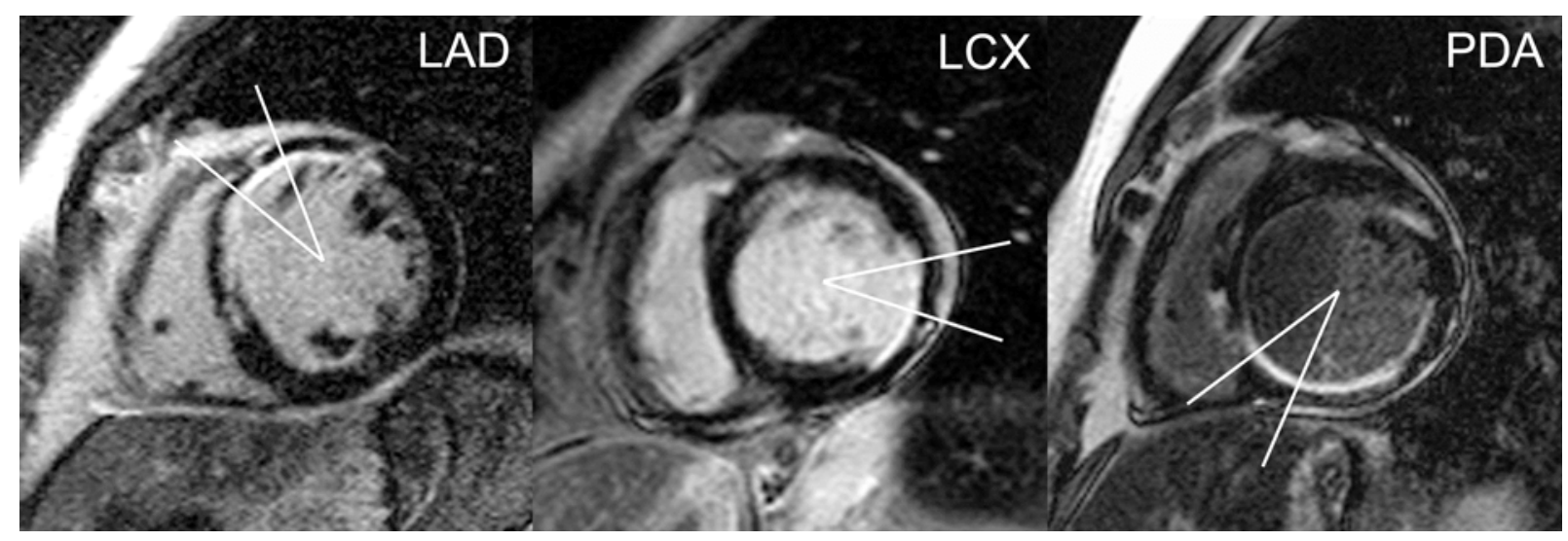

Fig. (1). Myocardial sectors by coronary artery of interest. Thirty-degree myocardial sectors straddle segments representative of the distribution of a particular coronary artery, with the coronary artery of interest centered over the respective sector. The myocardial sector is centered below the visualized LAD course in the anterior interventricular groove for LAD lesions (left), the mid-lateral free-wall (centered opposite the mid-interventricular septum) for lesions in the LCX (middle), or the visualized PDA course in the posterior interventricular groove for proximal lesions in either the source artery of the PDA or the proximal PDA itself (right). 
Table 1. Average Values for the Measured Variables in All Patients (All) and in Each of the Vessel Groups

\begin{tabular}{|c|c|c|c|c|}
\hline Gender & $26 \mathrm{M} / 8 \mathrm{~F}$ & $4 \mathrm{M} / 2 \mathrm{~F}$ & $3 \mathrm{M} / 1 \mathrm{~F}$ & $19 \mathrm{M} / 5 \mathrm{~F}$ \\
\hline Percent Diameter Stenosis & $75 \% \pm 26 \%$ & $73 \% \pm 23 \%$ & $72 \% \pm 32 \%$ & $75 \% \pm 27 \%$ \\
\hline Percent Area Stenosis & $87 \% \pm 15 \%$ & $89 \% \pm 11 \%$ & $85 \% \pm 18 \%$ & $87 \% \pm 16 \%$ \\
\hline Collateral Score & $1.2 \pm 1.2$ & $1.0 \pm 1.1$ & $0.8 \pm 1.0$ & $1.4 \pm 1.3$ \\
\hline Scar Area & $\begin{array}{l}33 \% \pm 34 \%(\mathrm{~B}) \\
31 \% \pm 35 \%(\mathrm{M})\end{array}$ & $\begin{array}{l}63 \% \pm 47 \%(\mathrm{~B}) \\
61 \% \pm 42 \%(\mathrm{M})\end{array}$ & $\begin{array}{l}28 \% \pm 32 \%(\mathrm{~B}) \\
35 \% \pm 42 \%(\mathrm{M})\end{array}$ & $\begin{array}{l}26 \% \pm 27 \%(\mathrm{~B}) \\
22 \% \pm 29 \%(\mathrm{M})\end{array}$ \\
\hline Transmurality & $\begin{array}{l}28 \% \pm 33 \%(\mathrm{~B}) \\
27 \% \pm 35 \%(\mathrm{M})\end{array}$ & $\begin{array}{l}58 \% \pm 45 \%(\mathrm{~B}) \\
55 \% \pm 45 \%(\mathrm{M})\end{array}$ & $\begin{array}{l}25 \% \pm 32 \%(\mathrm{~B}) \\
36 \% \pm 42 \%(\mathrm{M})\end{array}$ & $\begin{array}{l}22 \% \pm 26 \%(\mathrm{~B}) \\
19 \% \pm 27 \%(\mathrm{M})\end{array}$ \\
\hline
\end{tabular}

(B) Base, (M) Mid.

significant ( $<<0.01$ for each) but only moderate in strength: percent diameter stenosis versus scar area $(\mathrm{r}=0.53)$, versus transmurality $(\mathrm{r}=0.52)$, and versus patchiness $(\mathrm{r}=0.35)$; percent area stenosis versus scar area $(\mathrm{r}=0.53)$, versus transmurality $(\mathrm{r}=0.52)$, and versus patchiness $(\mathrm{r}=0.33)$.

Displays of scar area, transmurality, and patchiness as a function of percent diameter stenosis for all 34 patients are shown in Fig. (2) (pooled basal and mid-LV results); identical relationships were observed for percent area stenosis (not shown). Using $70 \%$ stenosis as a threshold for hemodynamic significance at rest, there were statistically significant differences in collateralization and scar parameters between patients with stenoses above and below this threshold, but with considerable overlap in the range of values (Table 2). Of note is the high prevalence and quantity of myocardial scarring found beyond less severe (i.e. $<70 \%$ ) stenoses and in the absence of clinical histories of corresponding acute infarction. Conversely, some patients with significant stenoses (70-100\%) exhibited little to no scar (Fig. (2)).

With patients grouped by collateral score, mean $( \pm$ standard deviation) percent diameter stenosis was $48 \% \pm 10 \%$ for collateral score $=0$, compared to $93 \% \pm 14 \%$ for collateral score $=1-3(p<0.01)$ (Fig. (2)). Mean scar area was 19\% $\pm 29 \%$ for patients with collateral score $=0$, compared to $41 \% \pm 35 \%$ for collateral score $=1-3(\mathrm{p}=0.01)$. Scar transmurality showed a similar pattern; mean transmurality was $15 \% \pm 29 \%$ for patients with collateral score $=0$ compared to $37 \% \pm 34 \%$ for collateral score $=1-3(\mathrm{p}=0.01)$. Finally, patchiness was $30 \% \pm 39 \%$ for collateral score $=0$ compared to $50 \% \pm 35 \%$ for collateral score $=1-3(\mathrm{p}=0.08)$.

Separated by gender, mean ( \pm standard deviation) percent diameter stenosis was $61 \% \pm 19 \%$ for females compared to $79 \% \pm 26 \%$ for males $(\mathrm{p}=0.13)$. There were no statistically significant differences between females and males in scar area, transmurality or patchiness. Mean scar area was $32 \% \pm$ $41 \%$ for females and $32 \% \pm 32 \%$ for males $(\mathrm{p}=0.81)$; transmurality was $30 \% \pm 41 \%$ for females, $27 \% \pm 32 \%$ for males ( $\mathrm{p}=0.74)$; lastly, patchiness was $41 \% \pm 40 \%$ for female patients and $42 \% \pm 37 \%$ for males $(\mathrm{p}=0.82)$.
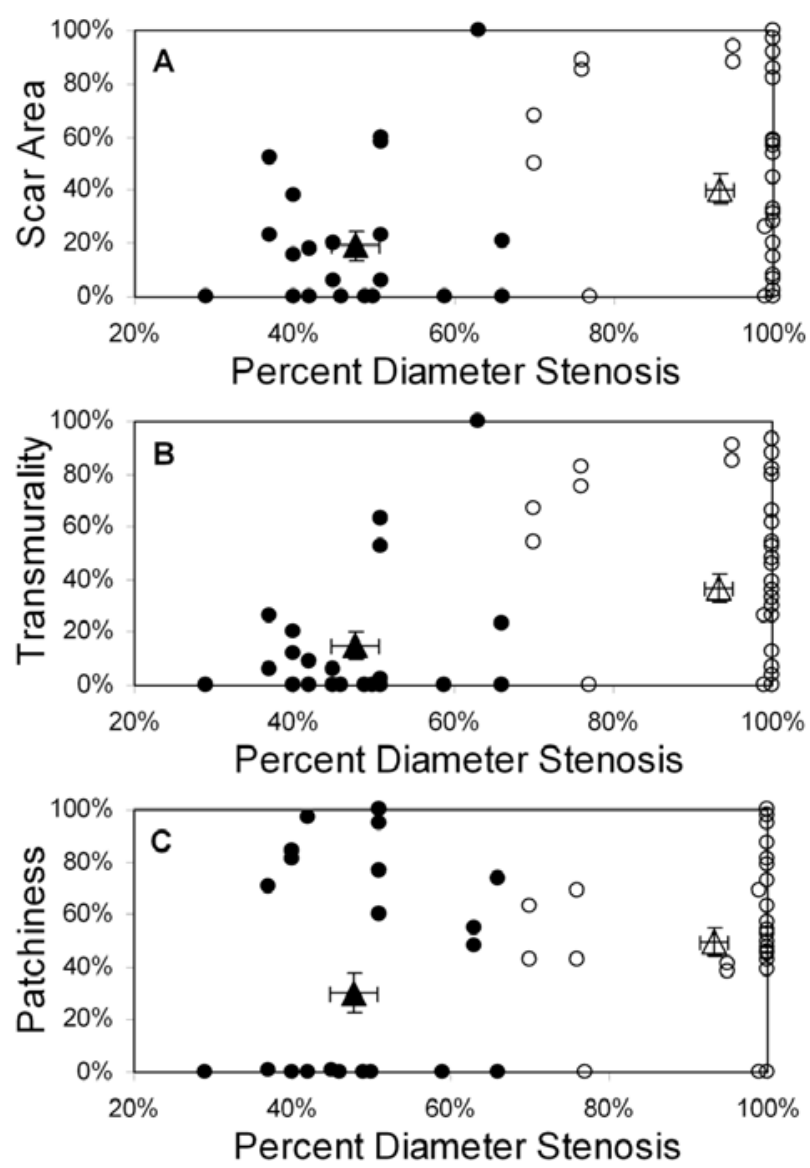

Fig. (2). Scar area (A), transmurality (B), and patchiness (C) as functions of percent diameter stenosis for all patients $(n=34)$; basal and mid-LV results are pooled. Patients are grouped by collateral score: score 0 (filled circles), score 1-3 (open circles). The filled triangle designates the mean value $( \pm$ SEM) for the 0 collaterals group and the open triangle designates the mean value $( \pm$ SEM) for the 1-3 collaterals group. Note that each scar parameter is computed within a $30^{\circ}$ sector defined in the appropriate coronary artery distribution for each vessel group. 


\section{Scar Area Model}

Gender, vessel, percent diameter stenosis, percent area stenosis and collateral score showed statistically significant influence on variability in the scar area model (Table $\mathbf{3}$ ). Using the model to predict scar area as a function of percent diameter stenosis for each of the statistically significant predictors (Fig. (3)), we found that female gender was associated with increased scar area at a given stenosis value. In addition, LAD index lesions resulted in more myocardial scar formation than LCX or PDA lesions (Fig. (3)), which were comparable. Lastly, the extent of collateralization was an important mitigating factor in scar development. Patients with collateral score $=0$ exhibited greater scar formation for a given stenosis level than those patients with Collateral Score $=1-2$ (Fig. (3)); furthermore, using this model, patients with collateral score $=3$ would not be expected to exhibit myocardial scarring until stenoses exceeded $70 \%$.

\section{Transmurality Model}

Gender and percent diameter stenosis showed statistically significant influence on variability in the transmurality model (Table 3); age and collateral score were of marginal statistical significance. Model-predicted transmurality as a function of percent diameter stenosis for the only statistically significant predictor, gender, is shown in Fig. (4).

\section{Patchiness Model}

None of the predictors was statistically significant in the patchiness model (Table 3); only percent diameter stenosis was of marginal statistical significance.

\section{DISCUSSION}

The relationship between the severity of coronary artery stenosis and resulting occult myocardial scarring in patients with chronic ischemic heart disease is not well understood. Our results demonstrate a high prevalence of significant myocardial scarring associated with coronary stenoses not considered angiographically significant. In addition, we found coronary collateralization and gender to be important mitigating factors in the extent of myocardial scar development.

DE-MRI imaging is well validated in the identification of chronic myocardial scar in animal models and clinical patients with high-grade stenosis $[18,19]$. Because of the high spatial resolution, transmural and subendocardial scar in the distribution of stenotic vessels can be differentiated [20,21]. The increase in myocardial scarring in the range of angiographically significant stenosis is consistent with hemodynamic data from animal models and mathematical simulations, demonstrating impaired flow at rest at a percent diameter stenosis of $75-85 \%$ [1-3]. In human studies, similar

Table 2. Effects of Hemodynamically Significant Stenoses

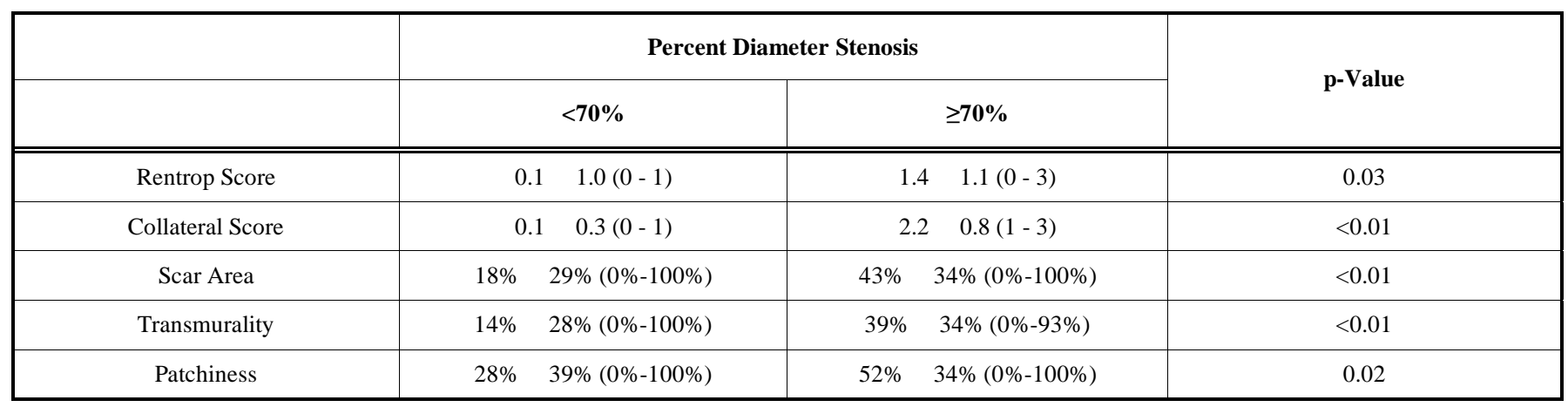

Data are presented as mean standard deviation (range)

P-values are the results of Student's t-test between groups.

Table 3. Statistical Significance of Predictor Variables for the Scar Area, Transmurality and Patchiness Linear Mixed Models

\begin{tabular}{|c|c|c|c|}
\hline & Scar Area & Transmurality & Patchiness \\
\hline \hline Gender & $<\mathbf{0 . 0 1}$ & $\mathbf{0 . 0 2}$ & 0.59 \\
\hline Age & 0.17 & 0.07 & 0.43 \\
\hline Vessel & $<\mathbf{0 . 0 1}$ & 0.46 & 0.39 \\
\hline LV Level & 0.84 & 0.62 & 0.21 \\
\hline \% Diameter Stenosis & $<\mathbf{0 . 0 1}$ & $<\mathbf{0 . 0 1}$ & 0.05 \\
\hline \% Area Stenosis & $\mathbf{0 . 0 4}$ & 0.10 & 0.46 \\
\hline Rentrop Score & 0.88 & 0.89 & 0.77 \\
\hline Collateral Score & $\mathbf{0 . 0 3}$ & 0.06 & 0.30 \\
\hline
\end{tabular}

Predictors that reached statistical significance $(\mathrm{p}<0.05)$ are shown in bold type. Predictors of marginal statistical significance $(0.05 \leq \mathrm{p}<0.10)$ are shown in italics. See text for detailed descriptions of the models. 
results were reproduced in patients with limited, focal disease when QCA was used [4].
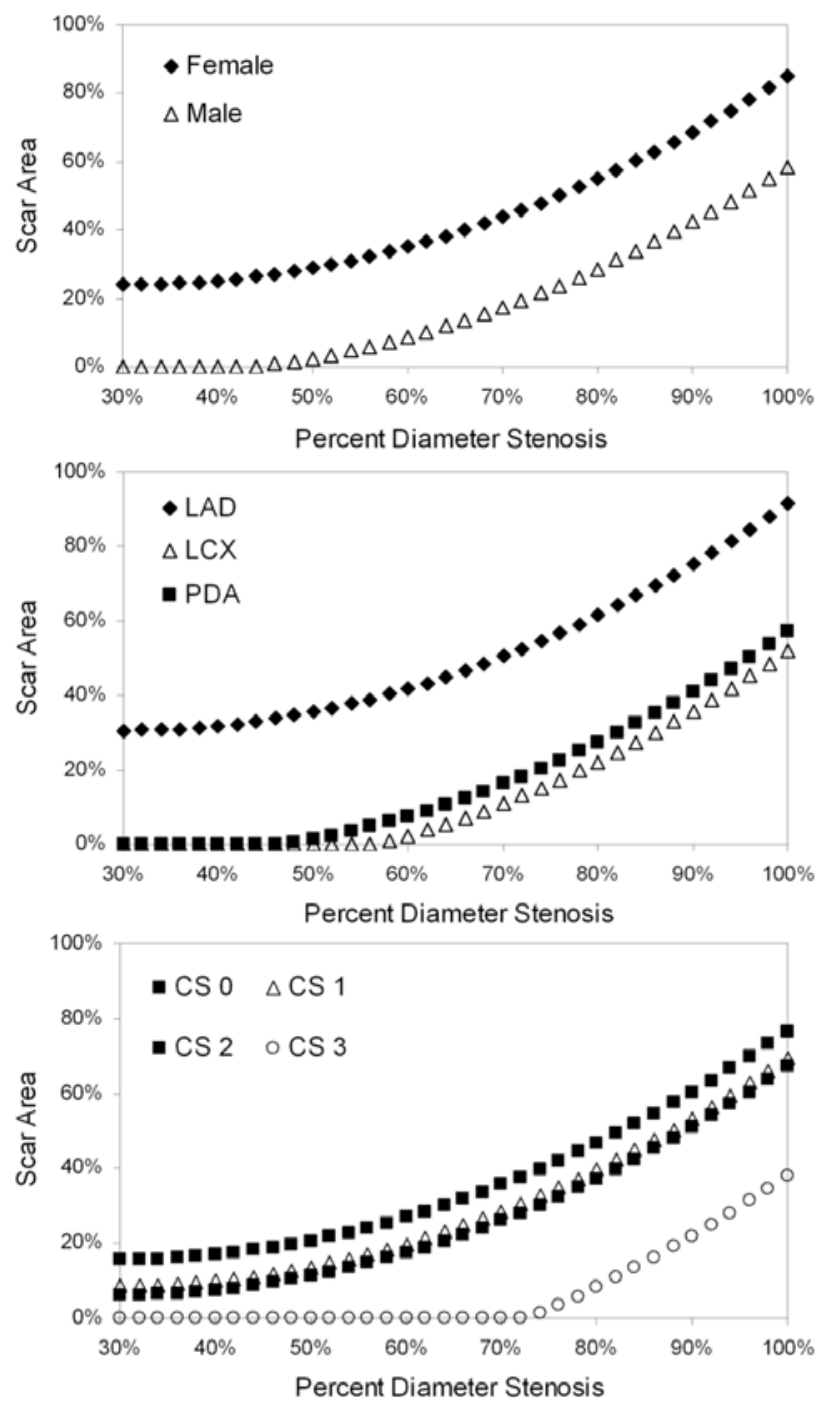

Fig. (3). Model-predicted scar area as a function of percent diameter stenosis. Each graph depicts a separate statistically significant predictor of variability in the scar area linear mixed-model (see text for details): gender, vessel containing the index lesion and collateral score (CS).

Also consistent with previous hemodynamic data is the significant influence of coronary collaterals. In the current study, collaterals were found only in those patients with a high degree of coronary stenosis (>70\%) [22]. Furthermore, collaterals shifted the stenosis-scar curve down and to the right. In patients without collaterals, myocardial scar was evident at angiographic stenosis of $30 \%$. However, with 3 sets of collaterals, scar did not appear until about $70 \%$ stenosis. These findings are consistent with clinical studies, showing that in patients with chronic stable CAD, well developed collateral vessels were associated with reduced infarct size $[23,24]$.

The extent of collaterallization may also explain differences in scar extent found between men and women. In our models, females consistently demonstrated a greater amount of myocardial scar compared to males over the range 30$100 \%$ stenosis. However, there was also a clear difference in the number of collateral vessels between these groups, with an average of only 0.5 sets of collaterals in female patients versus 1.5 sets of collaterals in male patients. The lower frequency of collaterals in women than in men has been noted by other investigators [25]. These results are important, as CAD is the leading cause of death in women and MRI imaging could play an importatnt role in the identification of subclinical disease in order to identify women for early preventive interventions [26].

An interesting result is the presence of scar in segments within the distribution of coronary arteries with mild stenosis. However, hemodynamic studies have demonstrated impaired flow reserve at percent diameter stenoses of $30-50 \%$ [1-4]. Such levels of stenosis could be associated with repeated episodes of ischemia and eventual scar formation. However, another explanation for such myocardial scar formation distal to mild stenoses implicates recent pathophysiologic descriptions of atherosclerotic lesion development [27]. Pathological studies suggest that atherosclerotic lesions progress through repeated episodes of plaque rupture, followed by healing. These episodes can cause repetitive episodes of transient coronary artery thrombotic stenosis/occlusion and/or peripheral thromboembolization leading to recurrent focal myocardial damage, reflected in scar along the distribution of distal vessel branches [28,29]. Using DEMRI, small areas of myocardial scarring have been observed after coronary intervention and bypass surgery, situations where local occlusion and peripheral embolization are common $[30,31]$.

\section{Limitations}

Our study has the following limitations. The study population was small and most of the included lesions were RCA lesions. The patients typically had advanced, multi-vessel CAD and frequently a history of myocardial infarction in remote segments. Clinical, hemodynamic studies in patients with advanced multi-vessel disease have typically shown a weak correlation between percent stenosis and hemodynamic significance [32,33]. In addition, concomitant information about myocardial ischemia in the examined segments was not available. Future studies in patients with 1-vessel disease and simultaneous hemodynamic assessment of lesion significance (e.g. fractional flow reserve CFR or stress testing) will provide further insights.

The analysis of DE-MRI images was restricted to a $30^{\circ}$ myocardial sector centered on the coronary artery of interest, which excluded some of the myocardium supplied by that artery. However, our intention was to exclude the effects of stenoses in branches of the coronary artery of interest and/or other coronary arteries, which could only be accomplished by specifying a smaller region of interest. A $45^{\circ}$ sector was also evaluated initially but was not found to provide advantages over the $30^{\circ}$ sector upon preliminary review of the data (results were unchanged).

\section{CONCLUSIONS}

Using QCA and DE-MRI, we demonstrate a significant relationship between the severity of proximal coronary artery stenosis and the extent of myocardial scar in the setting of 


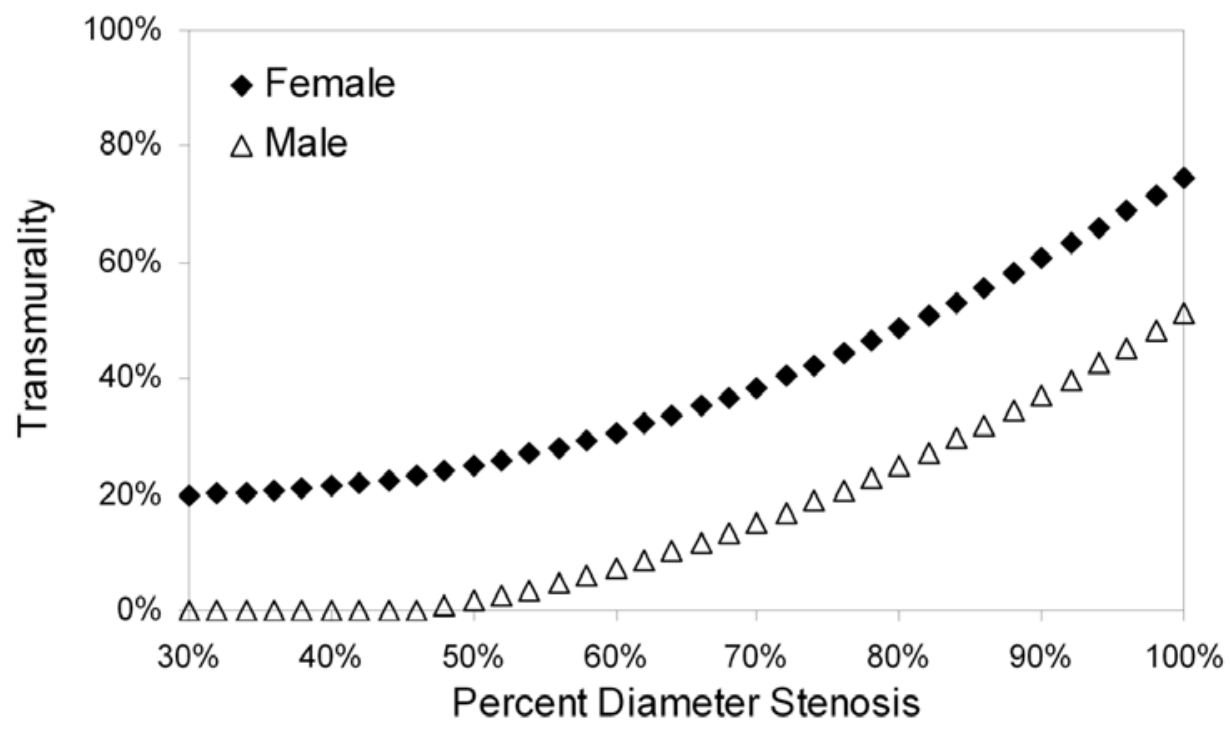

Fig. (4). Model-predicted transmurality as a function of percent diameter stenosis. Gender was the only statistically significant predictor of variability in the transmurality linear mixed-model (see text for details).

chronic ischemic heart disease. This relationship likely reflects increasing ischemia leading to scar formation in the range of angiographically significant stenosis. The influence of collaterals is reflected in the rightward shift of the curve (less scar tissue) with increasing number of collaterals. We also found prevalent myocardial scarring in the absence of significant coronary artery stenosis, supporting aggressive medical therapy to stabilize plaque, with or without revascularization, to prevent myocardial injury. Lastly, DE-MRI provides important information about the impact of coronary artery atherosclerosis on the myocardium beyond the involved vessel in patients being considered for revascularization therapy.

\section{REFERENCES}

[1] Folts JD, Gallagher K, Rowe GG. Hemodynamic effects of controlled degrees of coronary artery stenosis in short-term and longterm studies in dogs. J Thorac Cardiovasc Surg 1977; 73: 722-27.

[2] Gould KL, Lipscomb K, Hamilton GW. Physiologic basis for assessing critical coronary stenosis. Am J Cardiol 1974; 33: 87-94.

[3] Klopp EH, Gott VL. A simple model of the hemodynamic effects of a proximal coronary artery narrowing. Ann Thorac Surg 1975; 19: 309-312.

[4] Wilson RF, Marcus ML, White CW. Prediction of the physiologic significance of coronary arterial lesions by quantitative lesion geometry in patients with limited coronary artery disease. Circulation 1987; 75: 723-32.

[5] Maes A, Flameng W, Nuyts J, et al. Histological alterations in chronically hypoperfused myocardium. Correlation with PET findings. Circulation 1994; 90: 735-45.

[6] Nicklas JM, Becker LC, Bulkley BH. Effects of repeated brief coronary occlusion on regional left ventricular function and dimension in dogs. Am J Cardiol 1985; 56: 473-8.

[7] Geft IL, Fishbein MC, Ninomiya K, et al. Intermittent brief periods of ischemia have a cumulative effect and may cause myocardial necrosis. Circulation 1982; 66: 1150-3.

[8] Elsässer A, Schlepper M, Klövekorn WP, et al. Hibernating myocardium: an incomplete adaptation to ischemia. Circulation 1997; 96: $2920-31$

[9] Simonetti OP, Kim RJ, Fieno DS, et al. An improved MR imaging technique for the visualization of myocardial infarction. Radiology 2001; 218: 215-23.

[10] Setser RM, Bexell DG, O'Donnell TP, et al. Quantitative assessment of myocardial scar in delayed enhancement magnetic resonance imaging. J Magn Reson Imaging 2003; 18: 434-41.
[11] Schvartzman PR, Srichai MB, Grimm RA, et al. Nonstress delayed-enhancement magnetic resonance imaging of the myocardium predicts improvement of function after revascularization for chronic ischemic heart disease with left ventricular dysfunction. Am Heart J 2003; 146: 535-41.

[12] White RD. MR and CT assessment for ischemic cardiac disease. J Magn Reson Imaging 2004; 19: 659-75.

[13] Hermiller JB, Cusma JT, Spero LA, Fortin DF, Harding MB, Bashore TM. Quantitative and qualitative coronary angiographic analysis: Review of methods, utility, and limitations. Cathet Cardiovasc Diagn 1992; 25: 110-31.

[14] Gronenschild E, Janssen J, Tijdens F. CAAS II: A second generation system for off-line and on line quantitative coronary angiography. Cathet Cardiovasc Diagn 1994; 33: 61-75.

[15] Cerqueira MD, Weissman NJ, Dilsizian V, et al. Standardized myocardial segmentation and nomenclature for tomographic imaging of the heart: a statement for healthcare professionals from the Cardiac Imaging Committee of the Council on Clinical Cardiology of the American Heart Association. Circulation 2002; 105: 539-42.

[16] Gonzalez RC, Woods RE. In: Gonzalez RC, Ed. Digital image processing. Massachusetts, Addison-Wesley 1992; 41-42.

[17] Crowder MJ, Hand DJ. Analysis of Repeated Measures. $1^{\text {st }}$ ed. London: Chapman \& Hall; 1990.

[18] Kim RJ, Wu E, Rafael A, et al. The use of contrast-enhanced magnetic resonance imaging to identify reversible myocardial dysfunction. N Eng J Med 2000; 343: 1445-53.

[19] Mahrholdt H, Wagner A, Holly TA, et al. Reproducibility of chronic infarct size measurement by contrast-enhanced magnetic resonance imaging. Circulation 2002; 106: 2322-7.

[20] Wagner A, Mahrholdt H, Holly TA, et al. Contrast-enhanced MRI and routine single photon emission computed tomography (SPECT) perfusion imaging for detection of subendocardial myocardial infarcts: an imaging study. Lancet $2003 ; 361: 374-9$.

[21] Wu E, Judd RM, Vargas JD, Klocke FJ, Bonow RO, Kim RJ Visualization of presence, location, and transmural extent of healed Q-wave and non-Q-wave myocardial infarction. Lancet 2001; 357: 21-8.

[22] Rentrop KP, Thornton JC, Feit F, Van Buskirk M. Determinants and protective potential of coronary arterial collaterals as assessed by an angioplasty model. Am J Cardiol 1988; 61: 677-84.

[23] Billinger M, Kloos P, Eberli FR, Windecker S, Meier B, Seiler C. Physiologically assessed coronary collateral flow and adverse cardiac ischemic events: a follow-up study in 403 patients with coronary artery disease. J Am Coll Cardiol 2002; 40: 1545-50.

[24] Habib GB, Heibig J, Forman SA, et al. Influence of coronary collateral vessels on myocardial infarct size in humans. Results of phase I thrombolysis in myocardial infarction (TIMI) trial. The TIMI Investigators. Circulation 1991; 83: 739-46. 
[25] Johansson S, Bergstrand R, Schlossman D, Selin K, Vedin A, Wilhelmsson C. Sex differences in cardioangiographic findings after myocardial infarction. Eur Heart J 1984; 5: 374-81.

[26] Mieres JH, Shaw LJ, Arai A, et al. Role of noninvasive testing in the clinical evaluation of women with suspected coronary artery disease: Consensus statement from the Cardiac Imaging Committee, Council on Clinical Cardiology, and the Cardiovascular Imaging and Intervention Committee, Council on Cardiovascular Radiology and Intervention, American Heart Association. Circulation 2005; 111: 682-96.

[27] Burke AP, Kolodgie FD, Farb A, et al. Healed plaque ruptures and sudden coronary death: evidence that subclinical rupture has a role in plaque progression. Circulation 2001; 103: 934-40.

[28] Davies MJ. A macro and micro view of coronary vascular insult in ischemic heart disease. Circulation 1990; 82: II38-46.

[29] Topol EJ, Yadav JS. Recognition of the importance of embolization in atherosclerotic vascular disease. Circulation 2000; 101: 57080 .
[30] Ricciardi MJ, Wu E, Davidson CJ, et al. Visualization of discrete microinfarction after percutaneous coronary intervention associated with mild creatine kinase-MB elevation. Circulation 2001; 103 : 2780-3.

[31] Steuer J, Bjerner T, Duvernoy O, et al. Visualisation and quantification of peri-operative myocardial infarction after coronary artery bypass surgery with contrast-enhanced magnetic resonance imaging. Eur Heart J 2004; 25: 1293-9.

[32] White CW, Wright CB, Doty DB, et al. Does visual interpretation of the coronary arteriogram predict the physiologic importance of a coronary stenosis? N Engl J Med 1984; 310: 819-24.

[33] Harrison DG, White CW, Hiratzka LF, et al. The value of lesion cross-sectional area determined by quantitative coronary angiography in assessing the physiologic significance of proximal left anterior descending coronary arterial stenosis. Circulation 1984; 69: $1111-9$.

(C) Bexell et al.; Licensee Bentham Open.

This is an open access article licensed under the terms of the Creative Commons Attribution Non-Commercial License (http://creativecommons.org/licenses/by$\mathrm{nc} / 3.0 /$ ) which permits unrestricted, non-commercial use, distribution and reproduction in any medium, provided the work is properly cited. 